\title{
The margin of internal risk volume on atrial septal and ventricular septal based on electrocardiograph gating 4DCT
}

\author{
Qian $\mathrm{Li}^{1}$, Ying Tong ${ }^{2}$, Guanzhong Gong ${ }^{2}$, Yong Yin ${ }^{2}$, Yaping Xu ${ }^{1}$ \\ ${ }^{1}$ Department of Radiation Oncology, Shanghai Pulmonary Hospital, Tongji University School of Medicine, Shanghai, China; ${ }^{2}$ Department of Radiation \\ Physics, Shandong Cancer Hospital and Institute, Shandong First Medical University and Shandong Academy of Medical Sciences, Jinan, China \\ Contributions: (I) Conception and design: G Gong, Y Xu; (II) Administrative support: Y Xu, Y Yin; (III) Provision of study materials or patients: G \\ Gong, Y Yin; (IV) Collection and assembly of data: Q Li, Y Tong; (V) Data analysis and interpretation: Q Li, Y Tong; (VI) Manuscript writing: All \\ authors; (VII) Final approval of manuscript: All authors. \\ Correspondence to: Guanzhong Gong. Department of Radiation Physics, Shandong Cancer Hospital and Institute, Shandong First Medical University \\ and Shandong Academy of Medical Sciences, No. 440 Jiyan Road, Jinan, China. Email: gongguanzhong@163.com; Yaping Xu. Department \\ of Radiation Oncology, Shanghai Pulmonary Hospital, Tongji University School of Medicine, No. 507 Zhengmin Road, Shanghai, China. \\ Email: xuyaping1207@163.com.
}

Background: The aim of this study was to quantify the margin of internal risk volume (IRV) on the atrial
septum (AS) and ventricular septum (VS) based on electrocardiograph gating (ECG-gating) 4DCT.
Methods: Twenty patients were enrolled and received an ECG-gating 4DCT scan performed in breath-
hold, and CT images were reconstructed at 5\% intervals of the cardiac cycle for a total of 20 phases (0-95\%).
The contouring of the AS and VS were delineated in each phase, and the displacements and margin of the
AS and VS were calculated. We fused the total of the AS and VS (0-95\% phase), which were recorded as
$\mathrm{AS}_{20}$ and $\mathrm{VS}_{20}$. The margins were applied to the AS and VS in every phase and revised according to the cover
rate of $\mathrm{AS}_{20}$ and $\mathrm{VS}_{20}$. Results: (I) The margins of the AS and VS according to displacements in the left-right, cranio-caudal, and antero-posterior direction were $3 \mathrm{~mm}, 3 \mathrm{~mm}$, and $3 \mathrm{~mm}$; and $3 \mathrm{~mm}, 3 \mathrm{~mm}$, and $2 \mathrm{~mm}$, respectively. (II) The volume of $\mathrm{AS}_{20}$ was $(11.80 \pm 3.72) \mathrm{cm}^{3}$, which was 2.9 times larger than the maximum volume of the AS. The volume of $\mathrm{VS}_{20}$ was $(60.45 \pm 12.92) \mathrm{cm}^{3}$, which was 1.6 times larger than the maximum volume of the VS. (III) The emendatory margins of the AS and VS in the left-right, cranio-caudal, and antero-posterior direction were $7 \mathrm{~mm}, 10 \mathrm{~mm}$, and $7 \mathrm{~mm}$; and $5 \mathrm{~mm}, 3 \mathrm{~mm}$, and $4 \mathrm{~mm}$, respectively. The emendatory margins were added to the AS and VS, and the coverage rates were $(95.88 \pm 3.29) \%$ and $(95.24 \pm 2.54) \%$, respectively.

Conclusions: The margin of IRV on the AS and VS could cover the movement of AS and VS induced by heartbeat in the left-right, cranio-caudal, and antero-posterior direction respectively during thoracic radiotherapy.

Keywords: Electrocardiograph gating (ECG); atrial septum (AS); ventricular septum (VS); margin; internal risk volume (IRV)

Submitted Mar 24, 2021. Accepted for publication Apr 29, 2021.

doi: $10.21037 / \mathrm{atm}-21-1162$

View this article at: http://dx.doi.org/10.21037/atm-21-1162

\section{Introduction}

Radiation-induced heart disease (RIHD) often results from radiotherapy for thoracic malignant tumors such as breast, esophageal, and central lung cancer (1-4). RIHD may include acute pericarditis, coronary atherosclerosis, myocardial injury, cardiac conduction system abnormality, and heart valve disease (5) and the few studies investigating the pathogenesis of RIHD show cardiac conduction abnormality, arrhythmia, and intracardiac conduction block as its main manifestations (6). The effective protection of 
the atrial septum (AS) and ventricular septum (VS) could relieve the arrhythmia and conduction block seen in RIHD, as the cardiac conduction system is mainly located in the AS and VS (7-9). The electrocardiogram changes of atrial and ventricular septal defects depended on the size of the defect range, and giant defects are characterized by pronounced signs of right ventricular hypertrophy. The defect range is too large and shows obvious signs of right ventricular hypertrophy. There was a significant positive correlation between defect range with the amplitude and duration of $\mathrm{P}$ wave, $\mathrm{R}$ wave amplitude in lead $\mathrm{V} 1$ and the $\mathrm{S}$ wave amplitude in lead V5 (10). Movement of the AS and VS are in real-time induced by the heartbeat, while there is obvious deviation between dose-volume parameters and the delivery dose since conventional statical simulated 3D-CT images exclude the motion information. Electrocardiograph gating (ECG-gating) 4D-CT could eliminate the influence of the heartbeat and visualize cardiac substructure, which would allow clinicians to quantify the movement and calculate the margins of the AS and VS utilizing its dynamic images (11).

The ICRU62 reported internal target volume is defined as adding appropriate margins to the clinical target volume and takes into account the effect of physiological movements (peristalsis, heartbeat, and respiration) on tumor morphology and displacement (12). However, dose-volume parameters based on static images cannot authentically reflect the irradiation dose of organs at risk (OARs) during treatment delivery $(13,14)$. An appropriate margin should be given to the OARs considering physiological movement, which is vital for the specific protection and improving the accurate dose assessment of OARs. Thus, we propose the internal risk volume (IRV) as the margin of OARs which can cover the range of movement.

The uncertainty of movement is determined using the system error $(\Sigma)$ and random error $(\sigma)$, where $\Sigma$ is the deviation between each fraction and plan and $\sigma$ is the deviation between interfraction, respectively (15). McKenzie et al. (16) proposed that (I) adding the $1.3 \Sigma$ width to OARs could cover $90 \%$ the contour of OARs in each direction, and (II) adding the $0.5 \sigma$ width to OARs based on the $1.3 \Sigma$ margin ensured the irradiation dose of OARs conformed to dose restrain. Our research group completed a study of coronary artery bifurcation movement applying this formula in the early stages and obtained encouraging results.

In the present study, we investigated the movement of the AS and the VS induced by the heartbeat, and determined the IRV of the AS and VS.

We present the following article in accordance with the MDAR reporting checklist (available at http://dx.doi. org/10.21037/atm-21-1162).

\section{Methods}

\section{Patients and images}

We selected 20 patients in our treatment center from March 2015 to November 2016, of whom 11 were male and 9 were female. The median age of patients was 58 (range, 48-66) years old. All patients underwent a retrospective ECG-gating 4DCT scan performed in inspiratory breath-hold using Siemens SOMATOM Definition equipment and CT images were reconstructed at $5 \%$ intervals of the cardiac cycle for a total of 20 phases $(0-95 \%)$, where the interval was $0.5 \mathrm{~mm}$ and the thickness was $0.75 \mathrm{~mm}$. All procedures performed in this study involving human participants were in accordance with the Declaration of Helsinki (as revised in 2013). Ethical approval was obtained at institutional review board of Shanghai Pulmonary Hospital for this retrospective study (approval ID: K20-174Y). Informed consent was waived.

\section{$A S$ and VS contouring}

We contoured the AS and VS using MIM Maestro 6.7.6, and the window width/level was $400 / 40 \mathrm{HU}$. The contouring of the VS was from the left ventricle visible at its apex, and the AS was from the layer where the right ventricle joins the right atrium to the layer where the left atrium disappears completely in the cranio-caudal axis. Contouring of the AS and VS are shown in Figure 1.

\section{Data statistics}

\section{Displacement of the AS and VS}

We counted the coordinate values of the AS and VS in the left-right, cranio-caudal, and antero-posterior axis among each phase of the cardiac cycle and calculated the maximum displacement of the AS and VS in these three axes. The maximum displacement was the difference between the maximum and minimum coordinate values.

\section{Margins of the AS and VS}

The margins of the AS and VS were calculated according to the standards proposed by McKenzie et al. (16), and the formula was $1.3 \Sigma+0.5 \sigma$. We calculated and recorded the difference of coordinate values on the AS/VS between $0 \%$ phase and the other 19 phases, and its mean value $(M)$ and standard deviation 

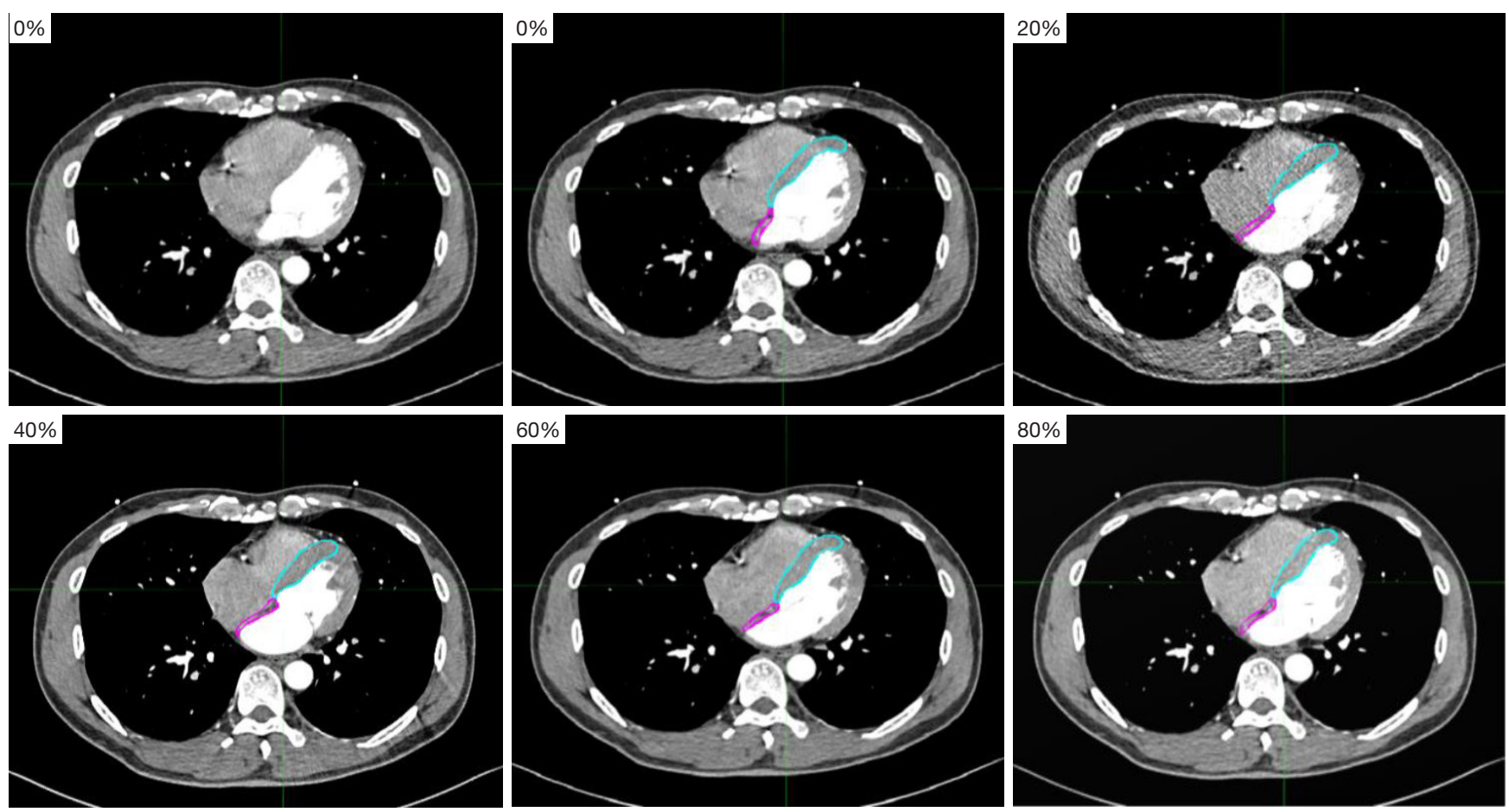

Figure 1 Contouring of the atrial septum (AS) and ventricular septum (VS). The figure shows the electrocardiograph gated (ECG-gated) 4D images of one patient (phase $0 \%, 20 \%, 40 \%, 60 \%, 80 \%$ ), and contouring of the AS and VS are shown in pink and blue, respectively.

(S) were then calculated. The standard deviation of $\mathrm{M}$ in all patients was $\Sigma$, and the root mean square of $S$ was $\sigma$.

\section{Volume of the AS and VS}

The volumes of the AS and VS in each phase during the cardiac cycle were recorded, and the change rate of AS/VS volume were calculated. This determined the ratio of the difference between the maximum and minimum volume and the minimum volume.

\section{Dice similarity coefficient (DSC) of the AS and VS}

The DSC of the AS/VS in the other 19 phases (5-95\%) were calculated based on the $0 \%$ phase. The formula was $D S C=2|A \cap B| /(|A|+|B|)$, where $A$ is the volume of the AS/VS at one of phases between $5-95 \%$, and $\mathrm{B}$ is the volume of the AS/VS at $0 \%$ phase.

\section{Emendatory margins of the AS and VS}

We fused the total of AS and VS (0-95\% phase) using MIM Maestro 6.7.6 workstation, which were recorded as $\mathrm{AS}_{20}$ and $\mathrm{VS}_{20}$. The margins were applied to the AS and VS in every phase, and revised according to the cover rate of $\mathrm{AS}_{20}$ and $\mathrm{VS}_{20}$. The emendatory margins were safe when the coverage rate exceeding $95 \%$. The coverage rate was defined as $\mathrm{C} \cap \mathrm{D} /$ $\mathrm{C}$, where $\mathrm{C}$ was the volume of $\mathrm{AS}_{20} / \mathrm{VS}_{20}$ contouring and $\mathrm{D}$ was the volume of emendatory margins added to AS/VS. The technical roadmap of this experiment is shown in Figure 2.

\section{Statistical methods}

We completed the data processing using SPSS19.0 software and expressed the results in the form of $\bar{x} \pm \mathrm{s}$. The sample data of AS and VS volume and DSC on the VS were normally distributed, and paired sample $t$ test was selected for data analysis. The sample data of DSC on the AS were not normally distributed, and the Wilcoxon test was then selected for data analysis. A P of less than 0.05 indicated a statistically significant difference.

\section{Results}

\section{The displacements of AS and VS}

The displacements of center of mass of the AS and VS in the left-right, cranio-caudal, and antero-posterior direction were $(5.04 \pm 1.99),(7.55 \pm 2.49),(6.68 \pm 1.60) ;(4.98 \pm 2.17)$, and $(3.95 \pm 1.05),(3.76 \pm 1.38) \mathrm{mm}$, respectively. The displacement 


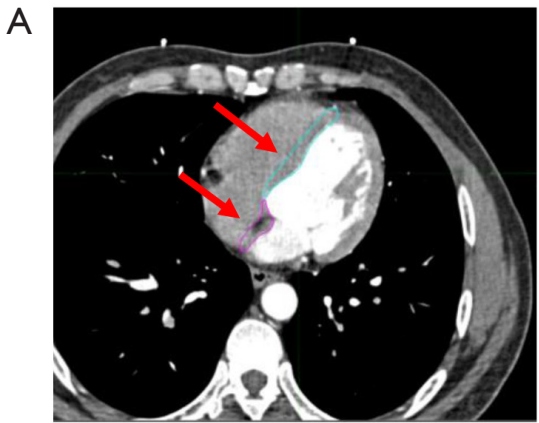

The contouring of AS and VS

C
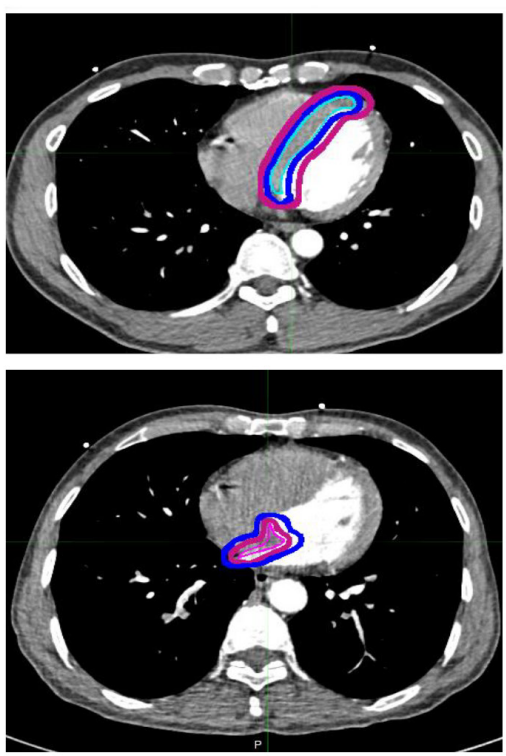
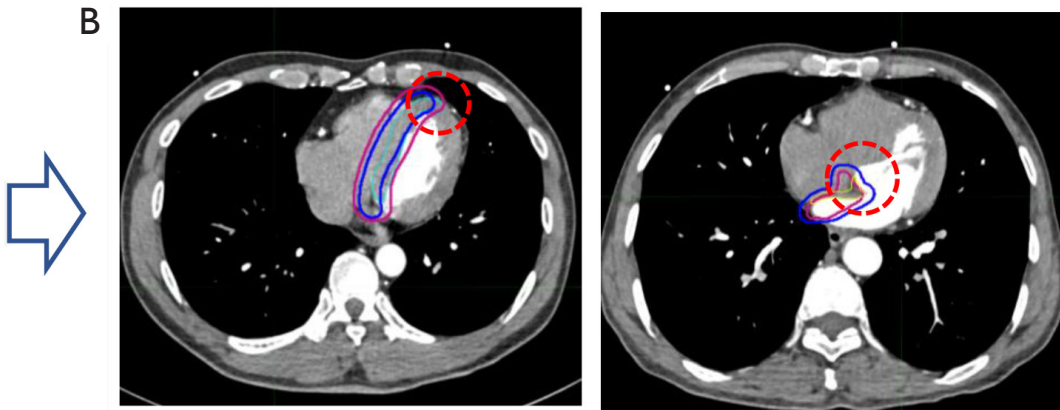

The calculated margin of AS and VS
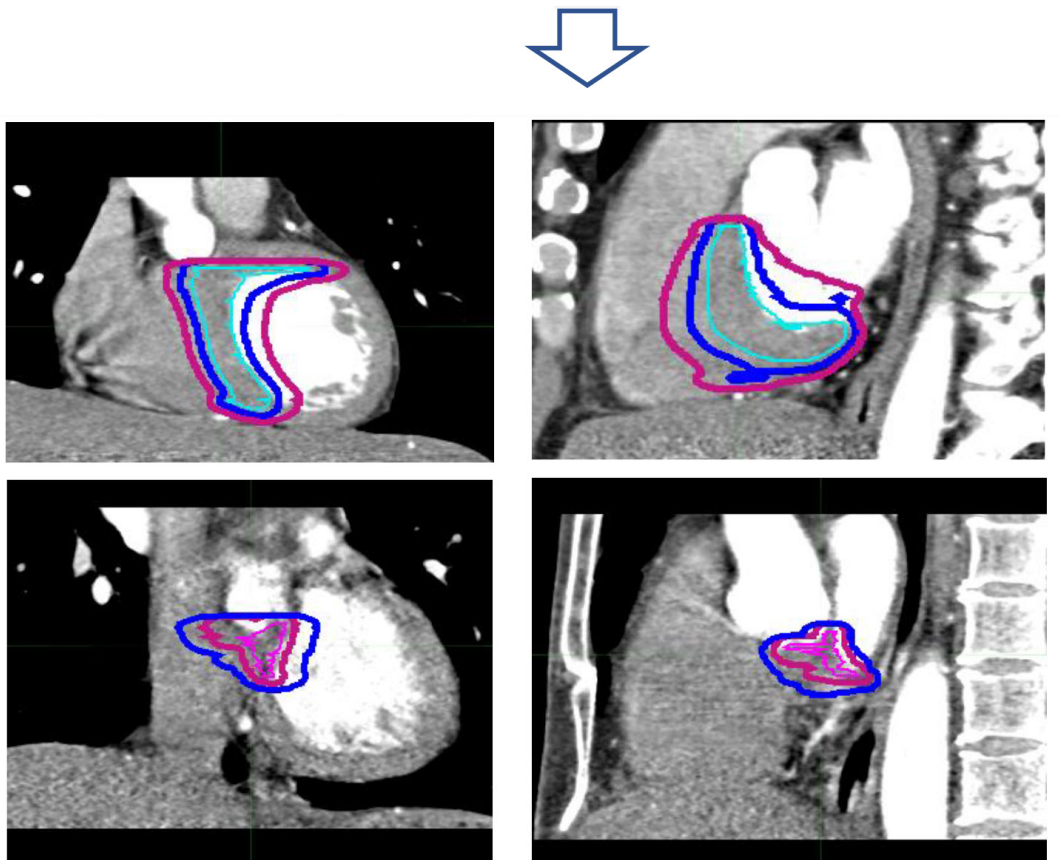

The emendatory margins of AS and VS

Figure 2 Technology roadmap. (A) The electrocardiograph gating (ECG-gating) 4DCT of one patient performed in inspiratory breathhold and contoured the atrial septum (AS) (pink line) and ventricular septum (VS) (blue line). (B) Margins of the AS (purple line) and VS (blue line) were calculated and applied, and the margins added to the AS and VS could not cover the AS \&VS completely in some phases (in the red circle area), which indicated that the calculated margin needed to be revised in some phases. (C) The emendatory margins of the AS and VS in cross-sectional, coronal, and sagittal planes. The light blue line is the VS, the dark blue line is the calculated margin of the VS, and the purple line is the emendatory margins of the VS. The pink line is the AS, the purple line is the calculated margin of the AS, and the dark blue line is the emendatory margins of the AS.

of the AS and VS in the left-right directions showed a mild difference, while the displacements of the VS in the craniocaudal and antero-posterior direction were greater than that of the VS.

\section{Margins of the AS and VS}

The margins of the AS and VS in the left-right, craniocaudal, and antero-posterior direction were 3,3 , and 3 ; and
3,3 , and $2 \mathrm{~mm}$, respectively.

\section{Volume variation of the AS and VS}

The average change rate of the AS and VS volume in the cardiac cycle were $61.43 \%$ and $38.98 \%$, and the maximum change rate reached to $147.06 \%$ and $64.93 \%$, respectively. The difference between the minimum and maximum volume of the AS and VS were both statistically significant $(\mathrm{P}<0.01)$. 
Table 1 Variation of the atrial septum (AS) and ventricular septum (VS) volume

\begin{tabular}{lccccc}
\hline Project & Minimum volume $\left(\mathrm{cm}^{3}\right)$ & Maximum volume $\left(\mathrm{cm}^{3}\right)$ & Change rate $(\%)$ & $P$ value & $\mathrm{t}$ value \\
\hline AS & $2.54 \pm 0.91$ & $3.98 \pm 1.29$ & $61.43 \pm 26.61$ & $<0.01$ & -11.847 \\
VS & $26.70 \pm 6.76$ & $36.72 \pm 7.99$ & $38.98 \pm 11.87$ & $<0.01$ & -16.412 \\
\hline
\end{tabular}

Table 2 Dice similarity coefficient (DSC) variation of the atrial septum (AS) and ventricular septum (VS)

\begin{tabular}{lccccc}
\hline Project & Minimum DSC & Maximum DSC & Change rate $(\%)$ & P value & t/z value \\
\hline AS & $0.081 \pm 0.067$ & $0.668 \pm 0.085$ & $1294.37 \pm 953.71$ & $<0.01$ & -4.107 \\
VS & $0.448 \pm 0.097$ & $0.878 \pm 0.044$ & $105.47 \pm 48.84$ & $<0.01$ & -19.435 \\
\hline
\end{tabular}

The variation of the AS and VS volumes are shown in Table 1.

\section{DSC variation of the AS and VS}

The average change rate of the AS and VS DSC in the cardiac cycle were $1,294.37 \%$ and $105.47 \%$, and the maximum change rate reached to $3,366.67 \%$ and $238.01 \%$, respectively. The difference between the minimum and maximum DSC of the AS and VS were both statistically significant $(\mathrm{P}<0.01)$. The variation of the AS and VS DSC are shown in Table 2.

\section{Emendatory margins of the AS and VS}

The volume of $\mathrm{AS}_{20}$ was $(11.80 \pm 3.72) \mathrm{cm}^{3}$ and was 4.6 times larger than the minimum volume and 2.9 times larger than the maximum volume of the AS, respectively. The volume of $\mathrm{VS}_{20}$ was $(60.45 \pm 12.92) \mathrm{cm}^{3}$ and was 2.3 times larger than the minimum volume and 1.6 times larger than the maximum volume of the VS, respectively. The emendatory margins of the AS and VS in the left-right, cranio-caudal, and antero-posterior direction were 7, 10, and 7; and 5, 3 , and $4 \mathrm{~mm}$, respectively. The emendatory margins were added to the AS and VS, and the coverage rates were $(95.88 \pm 3.29) \%$ and $(95.24 \pm 2.54) \%$, respectively. The calculated margins and emendatory margins applied to the AS and VS are shown in Figures 3 and 4.

\section{Discussion}

The difference between irradiation dose and dose-volume parameters during radiotherapy is caused by movement of the tumor and adjacent organs and proposes a challenge to the accurate prediction of efficacy and radiation-induced damage.
Adding the appropriate margin to target OARs covering the range of movement has been proposed as the primary solution in clinical applications (17). In a study published in the New England Fournal of Medicine, Cuculich et al. (13) reported the ablative dose delivered to the AS applying SBRT technology could significantly reduce the frequency of tachycardia and produce a beneficial effect on intractable arrhythmia. Thus, it is crucial for precise radiotherapy and protection of the AS and VS to explore the motion and margin of the AS and VS. In this study, we quantified the margins and IRV of the AS and VS based on ECG-gated 4DCT, which was beneficial to improving the accuracy of implementation and avoiding unnecessary exposure.

The motion tendency of the cardiac base and apex are heterochronous, as the whole heart twists during cardiac contraction, with the base rotating clockwise while the apex rotates counterclockwise (18). As movement of the cardiac base is more obvious than that of apex, displacement of the center of mass of the AS is in three directions and are greater than that of the VS. The VS is a concave structure attached to the left ventricle, and its movement is adduction towards the left ventricle in cardiac contraction, and abduction away from the left ventricle in cardiac diastole (19). Movement of the AS is more complex and involves movement towards the right atrium in the end-stage of cardiac contraction and the early-stage of diastole, then towards the left atrium, with mild motion in mid-diastole, and small displacement toward to left atrium movement followed by movement towards the right atrium instantly after the $\mathrm{P}$ wave (20). The overlapping volume of the AS in each phase and $0 \%$ time phase is small because of its small volume and complex motion, and results in a significant change rate of the DSC.

The margin formula proposed by McKenzie et al. (16) 

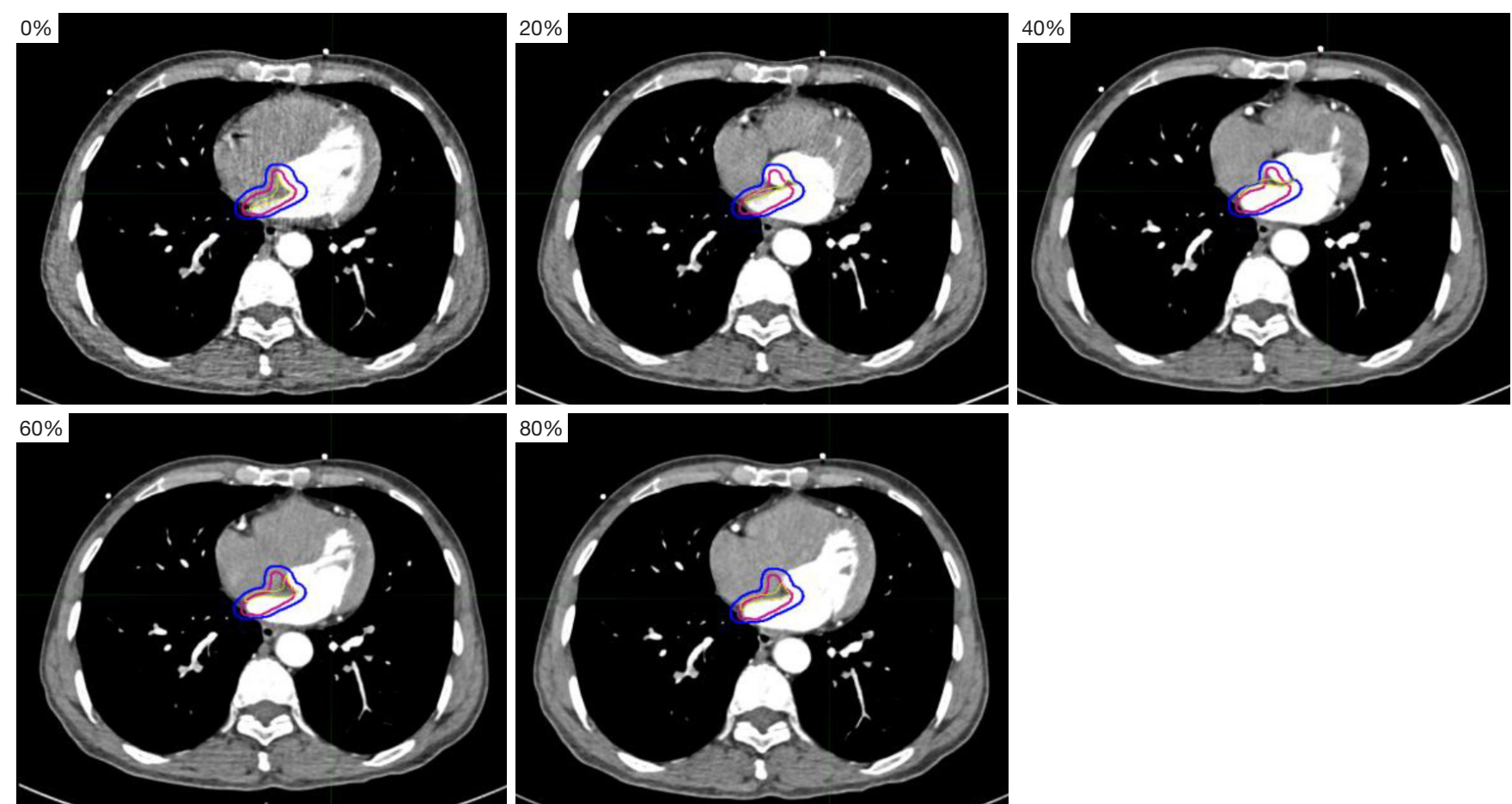

Figure 3 The calculated margins and emendatory margins applied to the atrial septum (AS). The yellow line represents the AS, the purple line is the calculated margins, and the dark blue line represents the emendatory margins.
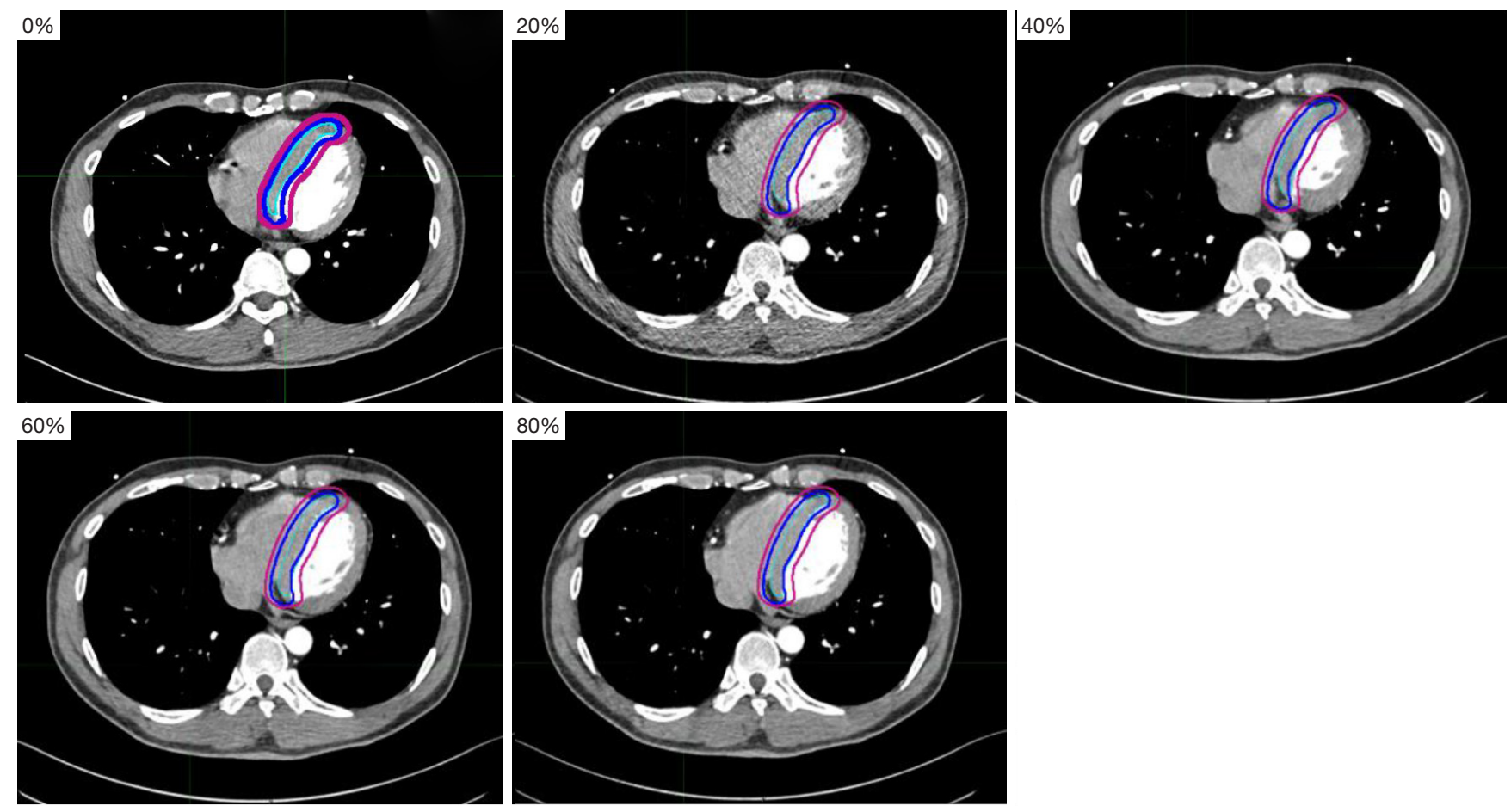

Figure 4 The calculated margins and emendatory margins applied to the ventricular septum (VS). The blue represents the VS, the dark blue line represents the calculated margins, and the purple line represents the emendatory margins. 
is only based on the displacement of OARs, while motion of the AS and VS depend on movement of the center of mass and the remarkable variation of volume and deformation. Accordingly, the remarkable variation explains the difference between the calculated margins and revised margins, and the emendatory margins of the AS in three directions were all greater than that of the VS. The same margins were added to the AS or VS and would have a different cover rate to the different volume and deformation between the VS and the AS. Therefore, the coverage effect of the VS could be significantly improved by adding 1$2 \mathrm{~mm}$ to the calculated margins. We added $4-7 \mathrm{~mm}$ to the calculated margins so the emendatory margins of the AS could reach a satisfactory cover rate for several reasons. Firstly, the error of contouring could not be ignored and the influence of cardiac contraction and diastolic effected on the AS was more significant, and secondly, the displacement of the AS was more obvious in the cranio-caudal direction, so its margin was the largest.

If the movement of tumor and organs were ignored during treatment planning, the target dose would be insufficient while the OARs dose would exceed the dose constraint, leading to a decline in the therapeutic effect (21). The $\mathrm{AS}_{20}$ and $\mathrm{VS}_{20}$ were the fusion of the AS and VS with 20 phases, respectively, and the contouring of $\mathrm{AS}_{20}$ and $\mathrm{VS}_{2}$ covered the motorial range of the AS and VS during the cardiac cycle, which was relatively safe and reliable. We found that the margins of the AS and VS (7, 10, and $7 \mathrm{~mm}$; and 5, 3, and $4 \mathrm{~mm}$ in the left-right, cranio-caudal and antero-posterior direction, respectively) could cover the motorial and deformable range of the AS and VS, so the margins of IRV were determined. However, further research is needed to explore whether the IRV could replace the OARs to participate in treatment planning and truly reflect the irradiation dose. Many scholars have quantified the movement of the cardiac substructure, such as the coronary artery and left ventricle, based on ECG-gated 4DCT, and proposed the concepts of IRV or planning risk volume $(15,18,22)$. Tong et al. (23) reported the variation of the dose-volume parameters $\left(\mathrm{D}_{\text {mean }}, \mathrm{V}_{20}, \mathrm{~V}_{30}\right.$, and $\left.\mathrm{V}_{40}\right)$ of the heart and left ventricular muscle induced by heartbeat during esophageal cancer radiotherapy. They found the parameters of the left ventricular muscle were significantly different between the maximum and minimum volume phase, and between the maximum and minimum DSC phase. This indicates volume and deformation were the main influencing factors on dose assessment.

The dual effect of the heartbeat and respiration can introduce complex changes to movement of the AS and VS when performed in free breath, and it is very difficult to separately analyze the two factors. Therefore, we used the cardiac systole and diastole while the patients held their breath as parameters in the present study. With the development of high dose rate radiotherapy technology, exposure time is no longer the main negative factor influencing radiotherapy. Breath-hold auxiliary radiotherapy is also increasingly applied to precise radiotherapy used in the treatment of breast, lung, and esophageal cancer, and the results of this study will be beneficial to the protection of the AS and VS during radiotherapy.

In summary, traditional methods of dose assessment are not precise, and do not favor the establishment of dose constraint on the AS and VS and the prediction of radiation-induced damage. The location, volume, and deformation of the AS and VS were significantly changed during the cardiac cycle, and the margin of IRV on the AS and VS were 7,10 , and $7 \mathrm{~mm}$; and 5, 3, and $4 \mathrm{~mm}$ in the left-right, cranio-caudal, and antero-posterior direction during thoracic radiotherapy.

\section{Acknowledgments}

Funding: None.

\section{Footnote}

Reporting Checklist: The authors have completed the MDAR reporting checklist. Available at http://dx.doi.org/10.21037/ atm-21-1162

Data Sharing Statement: Available at http://dx.doi. org/10.21037/atm-21-1162

Conflicts of Interest: All authors have completed the ICMJE uniform disclosure form (available at http://dx.doi. org/10.21037/atm-21-1162). The authors have no conflicts of interest to declare.

Ethical Statement: The authors are accountable for all aspects of the work in ensuring that questions related to the accuracy or integrity of any part of the work are appropriately investigated and resolved. All procedures performed in this study involving human participants were in accordance with the Declaration of Helsinki (as revised in 2013). Ethical approval was obtained at institutional review board of Shanghai Pulmonary Hospital for this retrospective study 
(approval ID: K20-174Y). Informed consent was waived. Open Access Statement: This is an Open Access article distributed in accordance with the Creative Commons Attribution-NonCommercial-NoDerivs 4.0 International License (CC BY-NC-ND 4.0), which permits the noncommercial replication and distribution of the article with the strict proviso that no changes or edits are made and the original work is properly cited (including links to both the formal publication through the relevant DOI and the license). See: https://creativecommons.org/licenses/by-nc-nd/4.0/.

\section{References}

1. Levis M, De Luca V, Fiandra C, et al. Plan optimization for mediastinal radiotherapy: Estimation of coronary arteries motion with ECG-gated cardiac imaging and creation of compensatory expansion margins. Radiother Oncol 2018;127:481-6.

2. Goody RB, O'Hare J, McKenna K, et al. Unintended cardiac irradiation during left-sided breast cancer radiotherapy. Br J Radiol 2013;86:20120434.

3. Ming X, Feng Y, Yang C, et al. Radiation-induced heart disease in lung cancer radiotherapy: A dosimetric update. Medicine (Baltimore) 2016;95:e5051.

4. Woodford K, Panettieri V, Ruben JD, et al. Limiting the risk of cardiac toxicity with esophageal-sparing intensity modulated radiotherapy for locally advanced lung cancers. J Thorac Dis 2016;8:942-9.

5. Boerma M, Sridharan V, Mao XW, et al. Effects of ionizing radiation on the heart. Mutat Res 2016;770:319-27.

6. Taunk NK, Haffty BG, Kostis JB, et al. Radiation-induced heart disease: pathologic abnormalities and putative mechanisms. Front Oncol 2015;18;5:39.

7. Mohan RA, Boukens BJ, Christoffels VM. Developmental Origin of the Cardiac Conduction System: Insight from Lineage Tracing. Pediatr Cardiol 2018;39:1107-14.

8. Burnicka-Turek O, Broman MT, Steimle JD, et al. Transcriptional Patterning of the Ventricular Cardiac Conduction System. Circ Res 2020;127:e94-106.

9. Ho SY, Sánchez-Quintana D. The importance of atrial structure and fibers. Clin Anat 2009;22:52-63.

10. Abdyzhaparova EK, Dzhishambaev ED, Usupbaeva DA. Electrocardiogram readings in different degrees of secondary atrial septal defect. Ter Arkh 2016;88:16-9.

11. Achenbach S, Ulzheimer S, Baum U, et al. Noninvasive coronary angiography by retrospectively ECG-gated multislice spiral CT. Circulation 2000;102:2823-8.

12. Brandner ED, Chetty IJ, Giaddui TG, et al. Motion management strategies and technical issues associated with stereotactic body radiotherapy of thoracic and upper abdominal tumors: A review from NRG oncology. Med Phys 2017;44:2595-612.

13. Cuculich PS, Schill MR, Kashani R, et al. Noninvasive Cardiac Radiation for Ablation of Ventricular Tachycardia. N Engl J Med 2017;377:2325-36.

14. John RM, Shinohara ET, Price M, et al. Radiotherapy for ablation of ventricular tachycardia: Assessing collateral dosing. Comput Biol Med 2018;102:376-80.

15. Stroom JC, Heijmen BJ. Limitations of the planning organ at risk volume (PRV) concept. Int J Radiat Oncol Biol Phys 2006;66:279-86.

16. McKenzie A, van Herk M, Mijnheer B. Margins for geometric uncertainty around organs at risk in radiotherapy. Radiother Oncol 2002;62:299-307.

17. Kataria T, Bisht SS, Gupta D, et al. Quantification of coronary artery motion and internal risk volume from ECG gated radiotherapy planning scans. Radiother Oncol 2016;121:59-63.

18. Nakatani S. Left ventricular rotation and twist: why should we learn? J Cardiovasc Ultrasound 2011;19:1-6.

19. Dwivedi A, Axel L. Abnormal Motion Patterns of the Interventricular Septum. JACC Cardiovasc Imaging 2017;10:1281-4.

20. Masai K, Kishima H, Takahashi S, et al. Interatrial septal motion as a novel index to predict left atrial pressure. Heart Vessels 2018;33:762-9.

21. Karava K, Ehrbar S, Riesterer O, et al. Potential dosimetric benefits of adaptive tumor tracking over the internal target volume concept for stereotactic body radiation therapy of pancreatic cancer. Radiat Oncol 2017;12:175.

22. Li Q, Tong Y, Yin Y, et al. Definition of the margin of major coronary artery bifurcations during radiotherapy with electrocardiograph-gated 4D-CT. Phys Med 2018;49:90-4.

23. Tong $Y$, Yin $Y$, Cheng $P$, et al. Impact of deformable image registration on dose accumulation applied electrocardiograph-gated 4DCT in the heart and left ventricular myocardium during esophageal cancer radiotherapy. Radiat Oncol 2018;13:145.

\section{(English Language Editor: B. Draper)}

Cite this article as: Li Q, Tong Y, Gong G, Yin Y, Xu Y. The margin of internal risk volume on atrial septal and ventricular septal based on electrocardiograph gating 4DCT. Ann Transl Med 2021;9(10):842. doi: 10.21037/atm-21-1162 